\title{
Blur Classification Using Wavelet Transform and Feed Forward Neural Network
}

\author{
Shamik Tiwari \\ Asst. Professor, FET, Mody Institute of Technology \& Science, Laxmangarh, India \\ Email: shamiktiwari@hotmail.com \\ V. P. Shukla \\ Professor, FET, Mody Institute of Technology \& Science, Laxmangarh, India \\ Email: drsvprasad2k@yahoo.com \\ S. R. Biradar \\ Professor, SDM College of Engineering, Hubli-Dharwad, India \\ Email: srbiradar@gmail.com \\ A. K. Singh \\ Asst. Professor, FET, Mody Institute of Technology \& Science, Laxmangarh, India \\ Email: ajay.kr.singh07@gmail.com
}

\begin{abstract}
Image restoration deals with recovery of a sharp image from a blurred version. This approach can be defined as blind or non-blind based on the availability of blur parameters for deconvolution. In case of blind restoration of image, blur classification is extremely desirable before application of any blur parameters identification scheme. A novel approach for blur classification is presented in the paper. This work utilizes the appearance of blur patterns in frequency domain. These features are extracted in wavelet domain and a feed forward neural network is designed with these features. The simulation results illustrate the high efficiency of our algorithm.
\end{abstract}

Index Terms-Blur, Motion, Defocus, Wavelet Transform, Neural Network.

\section{INTRODUCTION}

Barcode is the leading technology for automatic identification, which is used in a extensive range of realtime applications with a variety of codes [1]. In Comparison to 1-D barcode, 2-D barcode has high density, capacity, and reliability. Therefore, 2-D barcodes have been progressively more adopted these days. For example, a consumer can retrieve crucial information from the web page of the magazine or book, when he reads it, by just capturing the image of the printed QR code (2-D barcode) [2] related to URL. In addition to the URLs, 2-D barcodes can also symbolize visual tags in the supplemented real-world environment [3], and the adaptation from the individual profiles to 2D barcodes is usually exists [4]. Whereas, 1-D barcodes are traditionally scanned with laser scanners, 2-D barcode symbologies need imaging device for scanning.
Detecting bar codes from images taken by common used handheld devices like mobile camera is particularly challenging due to geometric distortion, noise, and blurring in image at the time of image acquisition. Image blurring is often an issue that affects the performance of a bar code identification system. Two most common types of blur are motion and defocus blur. Motion blur appears due to the relative motion among image capturing device and object at the time of image acquisition. Defocus blur appears due to the inaccurate focal length adjustment. Blurring induces the degradation of image quality. There are two approaches of deblurring; one is blind and other non-blind deconvolution. For blind restoration of barcode images it is essential to estimate blur parameters for deblurring. However, before application of any blur parameters estimation approach automatic blur classification is extremely desirable (i.e. to determine what type of blur is available in the acquired image).

Tong et al. [5] presented a method that applies Haar wavelet transform to estimate sharpness factor of the image to decide whether the image is sharp or blurred and then in case blurred image, they measure strength of blur. Yang et al. [6] addressed the motion blur detection scheme using support vector machine to classify the digital image as blurred or sharp. Aizenberg et al. [7] presented a work that identifies blur type, estimates blur parameters and perform image restoration using neural network. They considered four kinds of blur namely rectangular, motion, defocus, and Gaussian as a pattern classification problem. Bolan et al. [8] proposed an image blurred region detection and classification method which can automatically detect blurred image regions and blur type identification without image deblurring and kernel estimation. They developed a new blur metric 
using singular value feature to detect the blurred regions of an image and also analyze the alpha channel information and classify the blur type of the blurred image regions into defocus blur or motion blur, respectively. Renting et al. [9] presented a framework for partial blur detection and classification i.e. whether some portion of image is blurred as well as what types of blur arise in motion and defocus categories. They consider maximum saturation of color, gradient histogram span and spectrum details as blur features. Chakrabarti et al. [10] presented a method, which combines local frequency components, local gradient variations, and color channel information under the MRF segmentation framework to detect and extract motion blurred region in the image. Yan et al. [11] made an attempt to find a general feature extractor for common blur kernels with various parameters, which is closer to realistic application scenarios and applied deep belief networks for discriminative learning. Tiwari et al. [12] used statistical features of blur patterns in frequency domain and identified blur type with the use of feed forward neural network.

Wavelet features have been extensively used in object and texture classification. However, we have not found any application of wavelet features for blur classification. In this paper, we have proposed the use of wavelet features extraction of blur patterns in frequency spectrum and feed forward neural network for blur classification.

This paper is organized into seven sections including the present section. In section two to four, we discuss the theory of image degradation model, wavelet transform and feed forward neural network in that order. Section five describes the methodology of blur classification scheme. Section six discusses simulation results and in the final section seven, conclusion is discussed.

\section{IMAGE DEGRADATION MODEL}

The image degradation process in spatial domain can be modeled by the following convolution process [12]

$$
g(x, y)=f(x, y) * h(x, y)+\eta(x, y)
$$

where $g(x, y)$ is the degraded image, $f(x, y)$ is the uncorrupted original image, $h(x, y)$ is the point spread function that caused the degradation and $\eta(x, y)$ is the additive noise. Since, convolution in spatial domain is equivalent to the multiplication in frequency domain, equation (1) can be written as

$$
G(u, v)=F(u, v) H(u, v)+N(u, v)
$$

When the scene to be recorded translates relative to the camera at a constant velocity (vrelative) under an angle of $\theta$ radians with the horizontal axis during the exposure interval [0, texposure], the distortion is motion blur. Defining the length of motion as $L=v_{\text {relative }} \times$ $t_{\text {exposure, }}$ the point spread function (PSF) for uniform motion blur described as [12]

$$
h_{m}(x, y)=\left\{\begin{array}{c}
\frac{1}{L} \text { if } \sqrt{x^{2}+y^{2}} \leq \frac{L}{2} \text { and } \frac{x}{y}=-\tan \theta \\
0 \quad \text { otherwise }
\end{array}\right.
$$

The frequency response of PSF is called optical transfer function (OTF). The frequency response of $h_{m}$, is a SINC function given by

$$
H(u, v)=\operatorname{sinc}(\pi L(u \cos \theta+v \sin \theta))
$$

Fig. 1(a) and (b) show an example of motion blur PSF and corresponding OTF with specified parameters.

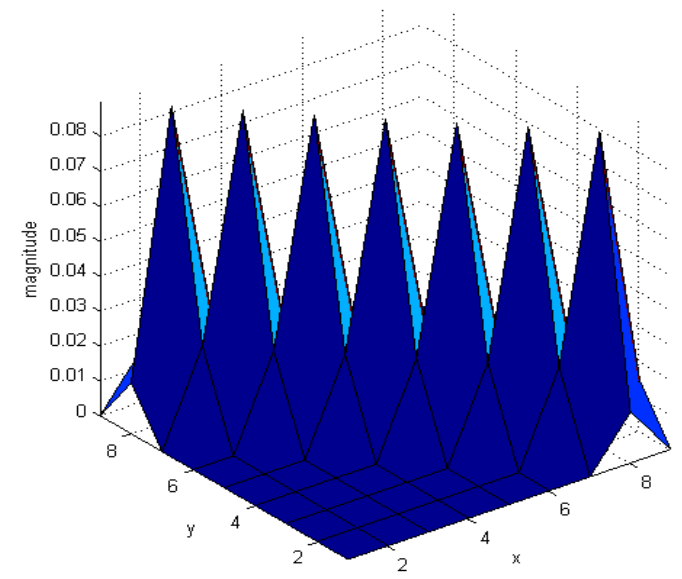

(a)

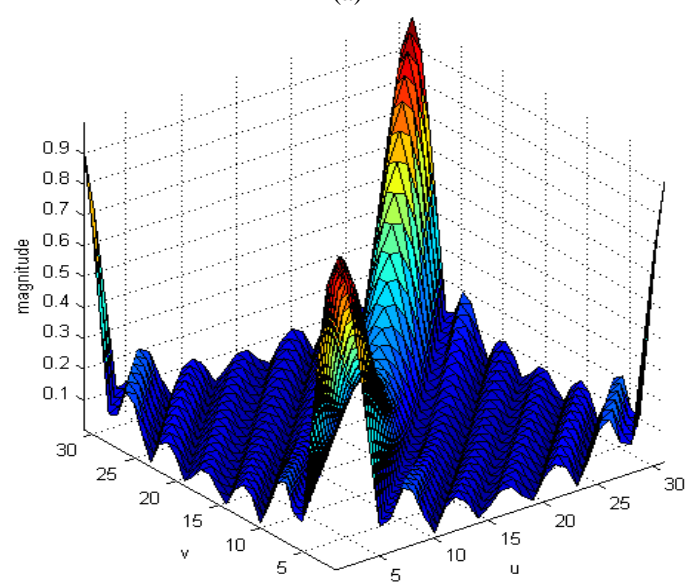

(b)

Fig1. (a) PSF of motion blur with angle 450 and length 10 pixels, (b) OTF of PSF in (a)

The out of focus blur caused by a system with circular aperture can be modeled as a uniform disk with radius $\mathrm{R}$ as [12]

$$
h_{d}(x, y)=\left\{\begin{array}{lc}
\frac{1}{\pi R^{2}} & \text { if } \sqrt{x^{2}+y^{2}} \leq R \\
0 & \text { otherwise }
\end{array}\right.
$$

The frequency response of equation (5) is given by (6), which is based on a Bessel function of the first kind [12]

$$
H(u, v)=\left[\frac{J_{1}\left(R \sqrt{u^{2}+v^{2}}\right)}{R \sqrt{u^{2}+v^{2}}}\right]
$$


where $J$ is the Bessel function of first kind and $R$ is radius of uniform disk. Fig.2 (a) and (b) show an example of PSF and corresponding OTF of defocus blur with specified radius.

The image degradation model for the presence of defocus and motion blur simultaneously in the image is given as [12]

$g(x, y)=f(x, y) * h_{d}(x, y) * h_{m}(x, y)+\eta(x, y)$

Since convolution is commutative, so joint blur PSF can be obtained as convolution of two blur functions as

$$
h(x, y)=h_{d}(x, y) * h_{m}(x, y)
$$

where $h_{d}(x, y)$ and $h_{m}(x, y)$ are point spread functions for motion defocus and blur respectively and ' $*$ ' is the convolution operator.

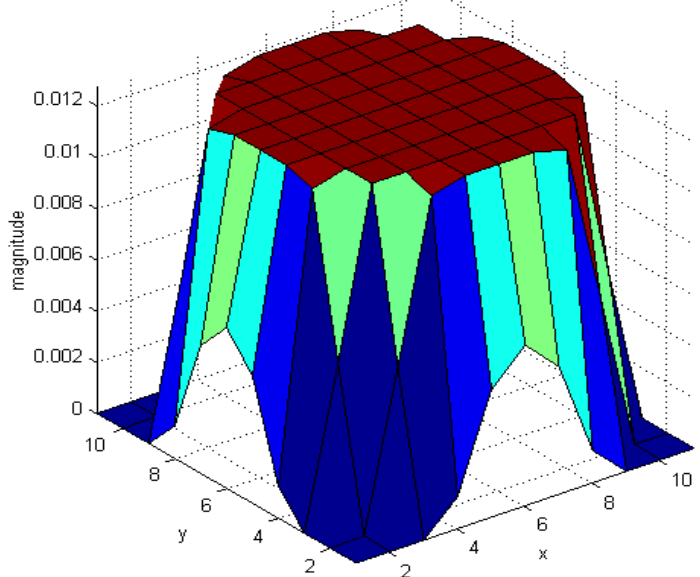

(a)

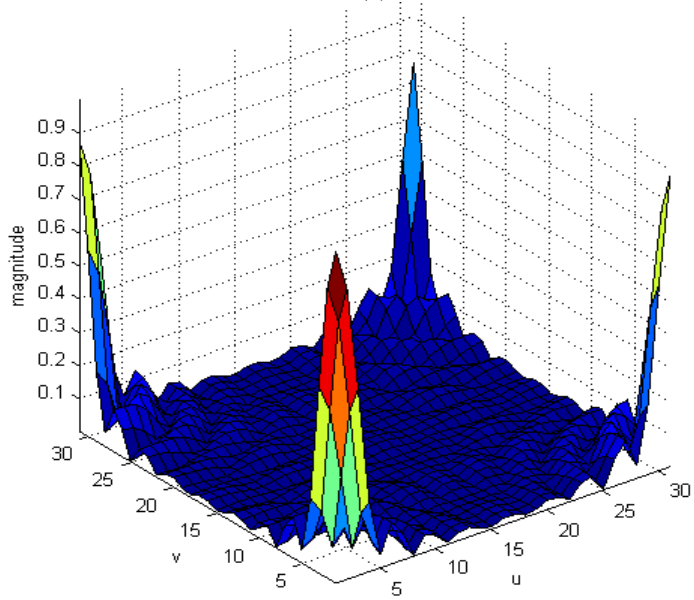

(b)

Fig2. (a) PSF of defocus blur with radius 5 pixels, (b) OTF of PSF in (a)

Fig.3(a) and (b) show an example of PSF and corresponding OTF of joint blur with specified parameters. This paper treats the blur effect caused by defocus and camera motion and combination of these two. This work does not consider additive noise in image degradation.

\section{WAVELET TRANSFORM}

The wavelet transform $[14,15]$ is a dominant and impressionable framework for image processing and analysis tasks. It is broadly used in the fields of image compression, segmentation, de-noising, recognition and fusion etc. The wavelet transform is based on the use of elementary functions, known as wavelets and scaling, which are produced by scaling and translation of a base function, called mother wavelet defined as

$$
\emptyset^{a, b}(x)=\frac{1}{\sqrt{a}} \emptyset\left(\frac{x-b}{a}\right)
$$

where ' $a$ ' is the scaling term and ' $b$ ' is the translation factor. In 2-D, it can be represented as

$$
\emptyset^{a, b}(x, y)=\frac{1}{\sqrt{a_{1} a_{2}}} \emptyset\left(\frac{x-b_{1}}{a_{1}}, \frac{x-b_{2}}{a_{2}}\right)
$$

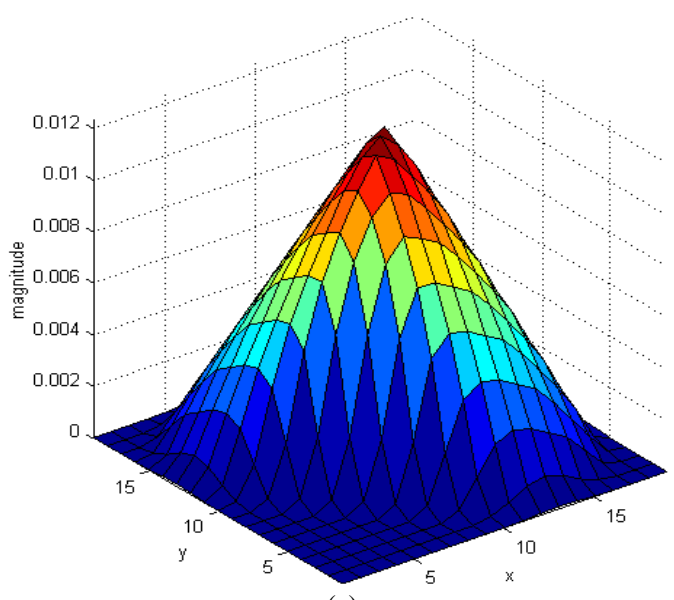

(a)

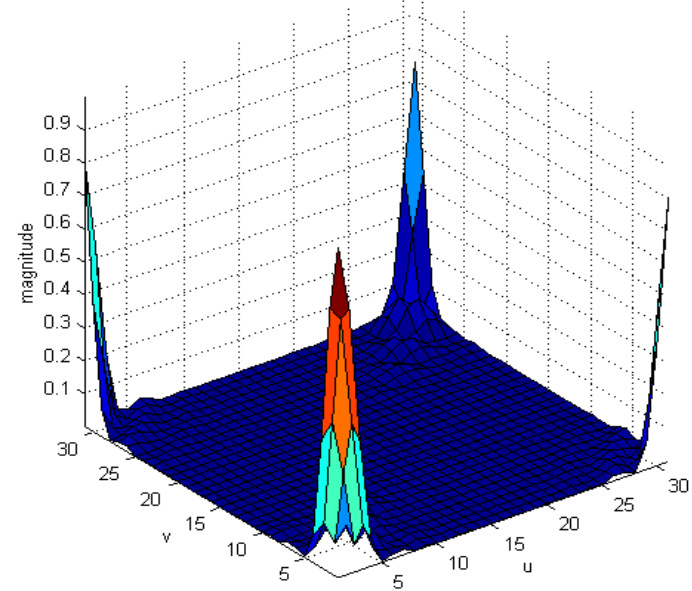

(b)

Fig3. (a) PSF of joint blur with angle $45^{\circ}$ and length 10 pixels, radius 5 pixels, (b) OTF of PSF in (a)

The wavelet transform of an image $f(x, y)$ is a set of coefficients $c_{i, j}$ that depend on the scale and shifting of the signal defined as

$$
c_{i, j}=\int_{-\infty}^{\infty} f(x, y) \emptyset^{a, b}(x, y) d x d y
$$


As a result of this, the wavelet transform decomposes the original image into a series of sub images with different levels. The two-dimensional discrete wavelet transform (DWT) hierarchically decompose an input image into a sequence of successively lower resolution images and their associated approximate and detail coefficient images. DWT is implemented by a set of filters, which are convolved with the image rows and columns. An image is convolved with low-pass and highpass filters and the odd samples of the filtered outputs are discarded, resulting in down sampling of the image by a factor of 2. The 1 level wavelet decomposition of an image $\mathrm{I}(\mathrm{x}, \mathrm{y})$ results in an approximation image $\mathrm{Xl}$ and three detail images $\mathrm{Hl}, \mathrm{Vl}$, and $\mathrm{Dl}$ in horizontal, vertical, and diagonal directions respectively. The features derived from these wavelet-transformed images are shown to be valuable for texture analysis [16]. Hiremath et al. [17] have discussed various significant characteristics of the wavelet transform based approaches for texture classification: (a) It is efficient to remove correlation available in the data and accomplish the similar objective as the linear transformation, (b) It gives direction sensitive information which is crucial in texture analysis, and (c) The computational complexity is appreciably reduced due to the wavelet decomposition.

We have considered blur patterns in frequency domain as textures and used this information to categorize the blur.

\section{FEED FORWARD NEURAL NETWORK}

An Artificial Neural Network (ANN) [12, 18] is a system, which is used to carry out classifications on datasets. The ANN can access a solution space that is not possible with the usual logical inferences based computational systems. In addition to this, it can operate on non-linear classification problems. They are composed of interconnected processing units called neurons that work as a collective unit. It can be used to establish complex relationships among inputs and outputs by identifying patterns in data. The feed forward neural network refers to the neural network which contains a set of source nodes which forms the input layer, one or more than one hidden layers, and single output layer. In case of feed forward neural network input signals propagate in one direction only, from input to output. There is no feedback path i.e. the output of one layer does not influence same layer. One of the best and widely recognized learning algorithms in training of multilayer feed forward neural networks is BackPropagation. The back propagation is a supervised learning algorithm, that receives sample of the inputs and related outputs to train the network, and then the error (difference between real and expected results) is calculated. The idea of the back propagation algorithm is

to minimize this error, until the neural network learns the training data.

\section{Methodology}

Blurring reduces sharp features of image such as boundaries, shape, regions, objects etc., which creates problem for further image analysis tasks. In spatial domain, it is not easy to differentiate the motion blur and defocus blur. However, these blurs have different appearance in frequency spectrum, which makes possible to categorize blurs. If we transform the blurred image in frequency spectrum, it can be seen from frequency spectrum of motion blurred image that the dominant parallel lines appear, that are orthogonal to the motion orientation with near zero values $[19,20]$. In defocused blur, we can see presence of some circular zero crossing patterns $[21,22]$ and in case of coexistence of both blurs joint effect becomes visible. Fig. 4 shows the effect of different blurs on the Fourier spectrum of original image. We propose the use of these frequency patterns as texture features for blur classification. The steps of the algorithm blur classification are given in fig.6. These are six major steps: image acquisition, preprocessing of images, computation of logarithmic frequency spectrum, feature extraction of blur patterns, data normalization, designing of neural network classifier system. In the initial steps we preprocessed image. Then, we have found the logarithmic frequency spectrum of blurred and non blurred image to get the blur patterns. Once the blur patterns acquired, the method demands the extraction of features. So the wavelet features have been calculated in the next step to prepare the training and testing database. Finally with this feature database training and testing performed using feed forward back propagation neural network.

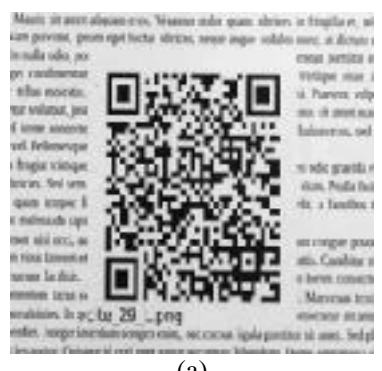

(a)

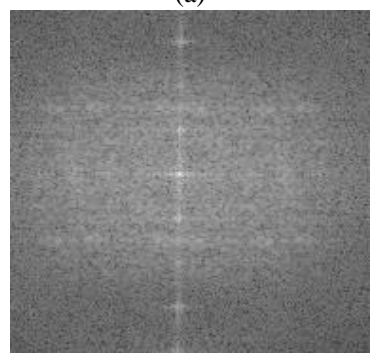

(c)

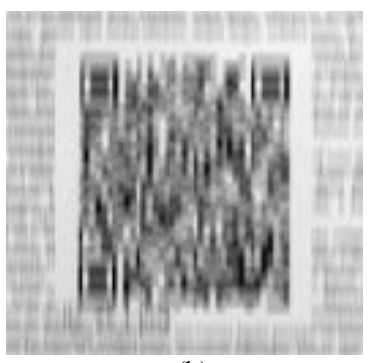

(b)

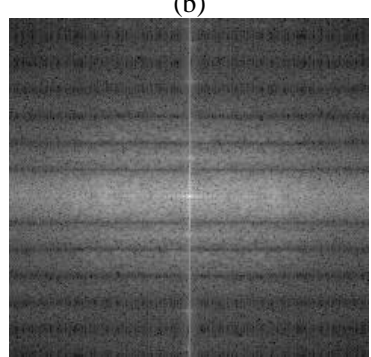

(d) 


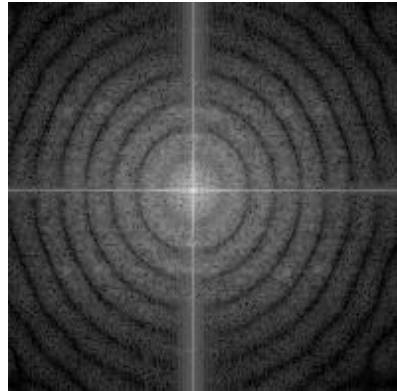

(e)

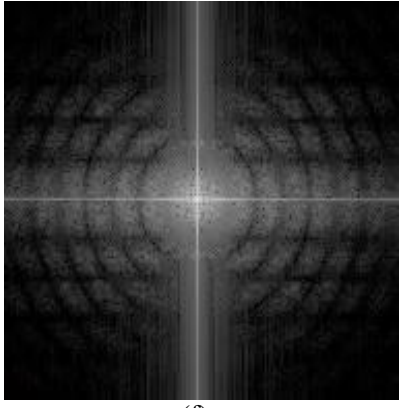

(f)
Figure 4: (a) Original image containing QR code [34] (b) Motion blurred image (c) Fourier spectrum of original image (d) Fourier spectrum of blurred image with motion length 15 pixels and motion orientation $90^{\circ}$ (e) Fourier spectrum of blurred image with defocus blur of radius 10 (f) Fourier spectrum of image with coexistence of both blurs

\section{A. Preprocessing}

Blurred image requires a number of preprocessing steps before feature extraction. First, the color image obtained by the digital camera is converted into an 8-bit grayscale image. This can be made by averaging the color channels or by weighting the RGB-parts according to the luminance perception of the human eye. The periodic transitions from one boundary of image to the next frequently lead to high frequencies, which are converted into visible vertical and horizontal lines in the power spectrum of image. Because these lines may distract from or even superpose the stripes caused by the blur, they have to be removed by applying a windowing function previous to frequency transformation. The Hanning window gives a fine trade-off between forming a smooth transition towards the image borders and maintaining enough image information in power spectrum. A 2-D Hann window of size N X M defined as the product of two 1D Hann windows:

$$
w[n, m]=\frac{1}{4}\left(1+\cos \left[2 \pi \frac{n}{N}\right]\right)\left(1+\cos \left[2 \pi \frac{m}{M}\right]\right)
$$

After that step, the windowed image can be transferred into the frequency domain by performing a fast Fourier transform. The power spectrum is calculated to facilitate the identification of particular features of the Fourier spectrum. However, as the coefficients of the Fourier spectrum decrease rapidly from its centre to the borders, it is difficult to identify local differences. Taking the logarithm of the power spectrum helps to balance this fast drop off. In order to obtain a centered version of the spectrum, its quadrants have to be swapped diagonally. In view of the fact that the remarkable features are around the centre of the spectrum, a centered portion of size $128 \times 128$ is cropped to perform wavelet transform, which reduces computation time. Images in fig. 5(a) to 5(d) show the results of hann windowing.

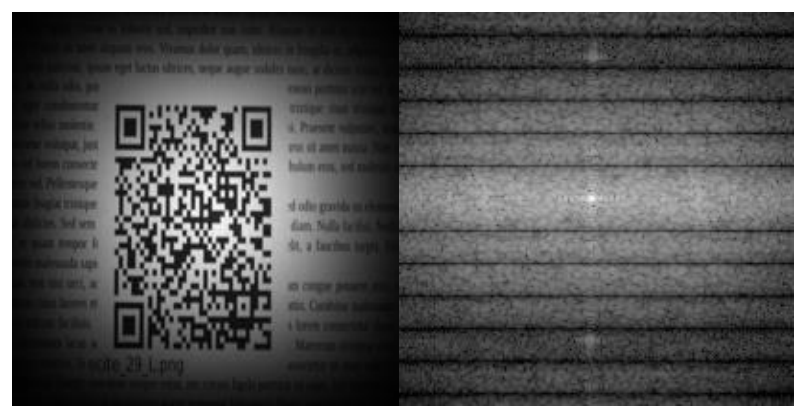

(a) (b)

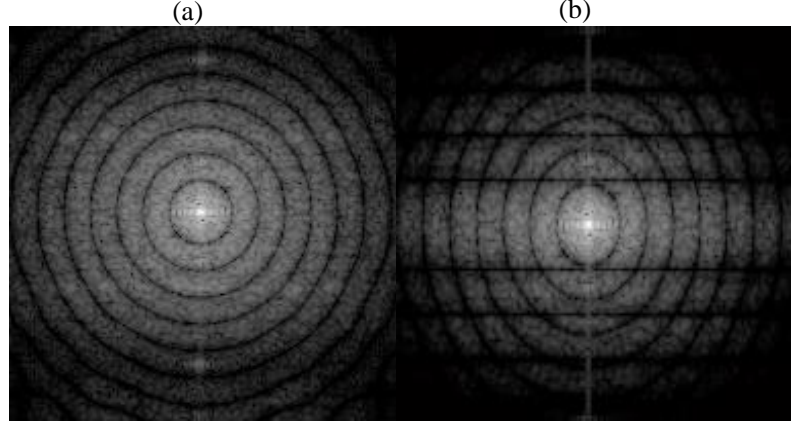

(c)

(d)

Figure 5: (a)-(d) Hann windowed images of images shown in figures 4(a), 4(d), 4(e) and 4(f) respectively.

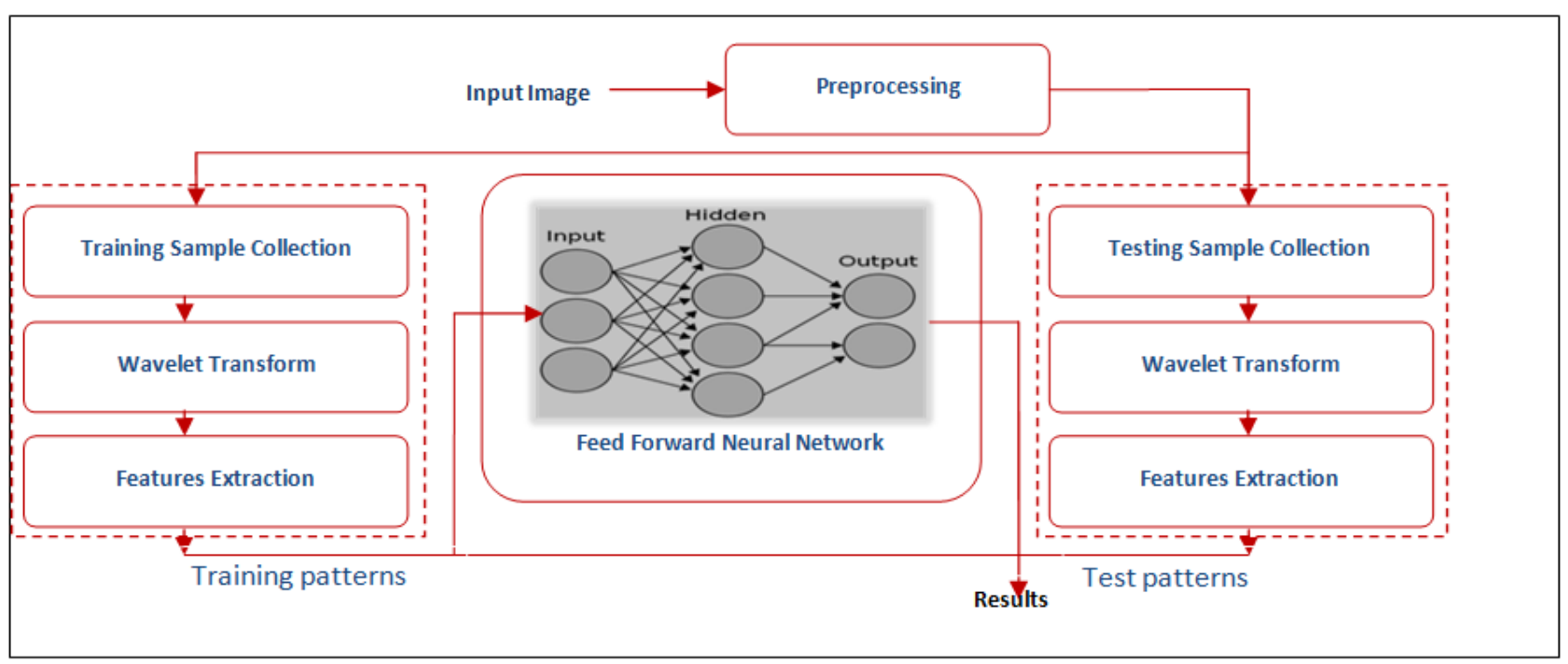

Figure 6: Blur classification framework 


\section{B. Feature Extraction}

Matlab Wavelet transforms toolbox used for wavelet transform with decomposition level 3. The Haar wavelet is chosen for its superior discriminating power. In comparison to other wavelets, the Haar wavelet is theoretically straightforward and precisely reversible without edge effects. The Haar transform does not have overlapping windows, which reflects only changes between adjacent pairs of pixels. These features make them ideal for the use in the wavelet transform. The wavelet energy gives the distribution of energy along the frequency axis over the scale and orientation of a detail image. Since most of the relevant texture information has been impartial by iteratively low-pass filtering, the energy of an approximation image is generally not considered as a feature. After achieving the wavelet transform the mean and standard deviation of the coefficients related with each decoposition is calculated. The mean of a detail image $\mathrm{I}_{\mathrm{i}}$ is calculated as:

$$
\mu_{i}=\frac{E_{i}}{M X N}
$$

where $M \times N$ is the size of detail image and $E_{i}$ is the energy of detail image. Energy is calculated by the sum of absolute values of wavelet coefficients.

$$
E_{i}=\sum_{\mathrm{x}=0}^{\mathrm{M}-1} \sum_{\mathrm{y}=0}^{\mathrm{N}-1}\left|\mathrm{I}_{\mathrm{i}}(\mathrm{x}, \mathrm{y})\right|
$$

The standard deviation of a detail image is calculated as:

$$
\sigma_{i}=\frac{\sqrt{\sum_{x=0}^{M-1} \sum_{y=0}^{N-1}\left(\left|I_{i}(x, y)\right|-\mu_{i}\right)^{2}}}{M X N}
$$

All these features are arranged in a manner such that the standard deviations remain in the first half of the feature vector and the means are arranged into the second half of the feature vector. So, we obtain a feature vector of size 18 consists of 9 mean energies and 9 standard deviations $f=\left(f_{1}, f_{2} \ldots, f_{18}\right)^{T}$ for each image. We have used this feature vector $f$ to categorize blurs.

\section{SIMULATION RESULTS}

The image database used for the simulation is the Brno Institute of Technology QR code image database [23]. 350 images from the database were considered. Then, the three different classes of blur i.e. motion, defocus and joint blur were synthetically introduced with different parameters to make the databases of 1050 blurred images (i.e., 350 images for each class of blur). A three layer Neural network was created with 18 nodes in the first (input) layer corresponding to size of input feature vector, 1 to 50 nodes in the hidden layer, and 3 nodes in the output layer (i.e. one node for each class). We have varied the number of nodes in the hidden layer in a simulation in order to determine the optimal number of hidden nodes. This was to avoid over fitting or under fitting the data. Finally, ten nodes in the hidden layer were selected to run the final simulation. The whole training and testing features set is normalized into the range of $[0,1]$, whereas the output class is assigned to zero for the lowest probability and one for the highest. During designing of neural network a cross-validation method is used to improve generalization ability. To perform the cross-validation procedure whole feature set is divided randomly into 3 sets training set, validation set and test set with a ratio $0.5,0.2$ and 0.3 respectively. The training set is used to learn the network. The validation set is used to validate the network i.e. to regulate network design parameters. The test set is used to test the generalization ability of the designed neural network

The aim of any classification technique is maximizing the number of correct classification given by True Positive Rate (TPR) and True Negative Rate (TNR), whereas minimizing the wrong classification given by False Positive Rate (FPR) and False Negative Rate. Terms True Positive (TP), True Negative (TN), False Positive (FP) and False Negative (FN) can be defined as below.

(i). True Positive $\left(T_{P}\right)$ : if test pattern is positive and it is predicted as positive, it is considered as a true positive.

(ii). True Negative (TN): if test pattern is negative and it is predicted as negative, it is considered as a true negative.

(iii). False Positive (FP): if test pattern is negative and it is predicted as positive, it is considered as a false positive.

(iv). False Negative ( $F N)$ : if test pattern is positive and it is predicted as negative, it is considered as a false negative.

In blur classification problem, we have used the four metrics for result evaluation as given in equations (16)(19).

$$
\begin{aligned}
& T_{\mathrm{PR}}=\frac{\text { Number of true positive }\left(\mathrm{T}_{\mathrm{P}}\right)}{\text { Total number of positive in dataset }\left(\mathrm{N}_{\mathrm{p}}\right)} \\
& \mathrm{T}_{\mathrm{NR}}=\frac{\text { Number of true negative }\left(\mathrm{T}_{\mathrm{N}}\right)}{\text { Total number of negative in dataset }\left(\mathrm{N}_{\mathrm{N}}\right)} \\
& \mathrm{F}_{\mathrm{PR}}=\frac{\text { Number of false positive }\left(\mathrm{F}_{\mathrm{P}}\right)}{\text { Total number of negative in dataset }\left(\mathrm{N}_{\mathrm{N}}\right)} \\
& \mathrm{F}_{\mathrm{NR}}=\frac{\text { Number of false negative }\left(\mathrm{F}_{\mathrm{N}}\right)}{\text { Total number of positive in dataset }\left(\mathrm{N}_{\mathrm{P}}\right)}
\end{aligned}
$$

The values of positive and Negative samples used as testing samples are 350 and 700, respectively for each blur categories. The data in Table 1 gives the above discussed rates given by the classifier. Classification accuracy for each blur class is calculated as mean of true positive rate and true negative rate. Testing results give 
classifications accuracies as $99.3 \%, 99.7 \%$, and $100 \%$ for motion, defocus and joint blur categories respectively. These classification accuracies show the high precision of our method.

Table 1: Blur Classification results

\begin{tabular}{|l|c|l|l|l|}
\hline & \multicolumn{4}{|c|}{ Classification Results(in percentage) } \\
\hline Blur Type & $\begin{array}{l}\text { True } \\
\text { Positive } \\
\text { Rate }\end{array}$ & $\begin{array}{l}\text { True } \\
\text { Negative } \\
\text { Rate }\end{array}$ & $\begin{array}{l}\text { False } \\
\text { Positive } \\
\text { Rate }\end{array}$ & $\begin{array}{l}\text { False } \\
\text { Negative } \\
\text { Rate }\end{array}$ \\
\hline Motion Blur & 98.6 & 100 & 0 & 1.4 \\
\hline Defocus Blur & 100 & 99.3 & 0.7 & 0 \\
\hline Joint Blur & 100 & 100 & 0 & 0 \\
\hline \multicolumn{6}{|c|}{ Motion blur classification accuracy 99.3 } \\
\hline \multicolumn{7}{|c|}{ Defocus blur classification accuracy 99.7 } \\
\hline \multicolumn{7}{|c|}{ Joint classification accuracy 100 } \\
\hline
\end{tabular}

\section{CONCLUSION}

In this paper, we have proposed a new blur classification scheme for barcode images taken by digital cameras. The blur type identification scheme utilizes the wavelet transform to discriminate blur patterns appear in frequency domain of blurred image, while considering them as texture features. This work classifies blur in motion, defocus and joint blur categories, which can further help to choose the appropriate blur parameter estimation approach for non-blind restoration of barcode images. In future this work can be extended by considering the noise in image degradation model.

\section{ACKNOWLEDGMENT}

We highly appreciate Faculty of Engineering and Technology, Mody University of Science \& Technology, Laxmangarh for providing facility to carry out this research work.

\section{REFERENCES}

[1] J. Vartiainen, T. Kallonen, and J. Ikonen, "Barcodes and Mobile Phones as Part of Logistic Chain in Construction Industry," The $16^{\text {th }}$ International Conference on Software, Telecommunication, and Computer Networks, pp. $305-$ 308, September 25-27, 2008.

[2] ISO/IEC 18004:2000. Information technology-Automatic identification and data capture techniques-Bar code symbology-QR Code, 2000.

[3] J. Rekimoto and Y. Ayatsuka, "Cybercode: Designing Augmented Reality Environments with Visual Tags," Proceedings of ACM International Conference on Designing Augmented Reality Environments, pp. 205215, 2000.

[4] T. S. Parikh and E. D. Lazowska, "Designing An Architecture for Delivering Mobile Information Services to the Rural Developing World," Proceedings of ACM International Conference on World Wide Web WWW, p.p. $54-62,2006$
[5] H. Tong, M. Li, H. Zhang, and C. Zhang, "Blur Detection For Digital Images Using Wavelet Transform," Proceedings of IEEE International Conference on Multimedia and Expo, Vol. 1, pp. 17-20, 2004.

[6] Kai-Chieh Yang, Clark C. Guest and Pankaj Das, "Motion Blur Detecting by Support Vector Machine," Proc. SPIE, pp. 5916-59160R, 2005.

[7] I. Aizenberg, N. Aizenberg, T. Bregin, C. Butakov, E. Farberov, N. Merzlyakov, and O. Milukova, "Blur Recognition on the Neural Network based on MultiValued Neurons," Journal of Image and Graphics. Vol.5, pp. 12-18, 2000.

[8] Su Bolan, Lu Shijian, and Tan Chew Lim, "Blurred Image Region Detection and Classification," In Proceedings of the 19th ACM international Conference on Multimedia (MM '11), pp. 34-40, 2011.

[9] Liu Renting, Li Zhaorong, Jia Jiaya, "Image Partial Blur Detection and Classification," IEEE Conference on Computer Vision and Pattern Recognition, pp.1-8, 2008.

[10] Ayan Chakrabarti, Todd Zickler, and William T. Freeman, "Correcting Over-Exposure in Photographs," in proc. 2010 IEEE conf. on Computer Vision and Pattern Recognition, pp. 2512-2519, 2010.

[11] R. Yan and L. Shao, "Image Blur Classification and Parameter Identification Using Two-stage Deep Belief Networks", British Machine Vision Conference (BMVC), Bristol, UK, 2013.

[12] Shamik Tiwari, V. P. Shukla, S. R. Biradar, Ajay Kumar Singh, " Texture Features based Blur Classification in Barcode Images," I.J. Information Engineering and Electronic Business, MECS Publisher, vol. 5, pp. 34-41, 2013.

[13] Shamik Tiwari, Ajay Kumar Singh and V. P. Shukla, "Certain Investigations on Motion Blur Detection and Estimation," Proceedings of International Conference on Signal, Image and Video Processing, IIT Patna, pp.108114, 2012.

[14] B. Kara and N. Watsuji, "Using Wavelets for Texture Classification," IJCI Proceedings of International Conference on Signal Processing, pp. 920-924, September 2003

[15] I. Daubechies, "Wavelet Transforms and Orthonormal Wavelet Bases, Different Perspectives on Wavelets," Proceedings of the Symposia in Applied Mathematics, vol. 47, pp. 1-33, American Mathematical Society, San Antonio, Tex, USA, 1993

[16] G. Van de Wouver, P. Scheunders, and D. Van Dyck, "Statistical Texture Characterization From Discrete Wavelet Representation," IEEE Trans. Image Process., vol. 8(11), pp. 592-598,1999.

[17] P. S. Hiremath, S. Shivashankar, "Wavelet Based Features for Texture Classification," GVIP Journal, vol. 6(3), pp. 55-58, 2006.

[18] Shamik Tiwari, Ajay Kumar Singh and V. P. Shukla, "Statistical Moments based Noise Classification using Feed Forward Back Propagation Neural Network," International Journal of Computer Applications, vol. 18(2), pp.36-40, 2011.

[19] Mohsen Ebrahimi Moghaddam, and Mansour Jamzad, "Linear Motion Blur Parameter Estimation in Noisy Images Using Fuzzy Sets and Power Spectrum Images," EURASIP Journal on Advances in Signal Processing, Vol. 2007, pp. 1-9, 2007.

[20] Michal Dobeš, Libor Machala, and Tomáš Fürst, "Blurred Image Restoration: A Fast Method of Finding the Motion Length and Angle," Digital Signal Processing, Vol. 20(6), pp. 1677-1686, 2010. 
[21] M. E. Moghaddam, "A Mathematical Model to Estimate Out of Focus Blur," Proceedings of 5th IEEE international symposium on Image and Signal Processing and Analysis, pp. 278-281, 2007.

[22] M. Sakano, N. Suetake, and E. Uchino, "A Robust Point Spread Function Estimation for Out-of-Focus Blurred and Noisy Images Based on a Distribution of Gradient Vectors on the Polar Plane," Journal of Optical Society of Japan, co-published with Springer-Verlag GmbH, Vol. 14(5), pp. 297-303, 2007.

[23] I. Szentandrási, M. Dubská, and A. Herout, "Fast Detection and Recognition of QR codes in HighResolution Images," Graph@FIT, Brno Institute of Technology, 2012.

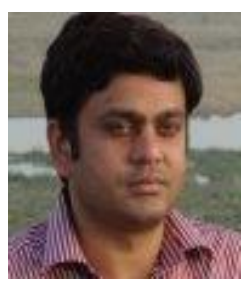

Shamik Tiwari has received his B.E. (Computer Sc. \& Engineering) in 2003, M.Tech. (Computer Sc. \& Engg.) in 2007 from RGPV University Bhopal and Dr. B. R. Ambedkar University Agra respectively. He has joined as an Asst. Professor in Mody Institute of Technology \& Science, Deemed University Laxmangarh in 2009. Presently, he is pursuing Ph.D. in Computer Sc. \& Engg. from the MITS Lakshmangarh. He has published over 25 papers in refereed journals and conference proceedings. He is an author of the book "Digital Image Processing” from Dhanpat Rai Publishing (India), His current research interest includes digital image processing, pattern classification, and their applications in computer vision.

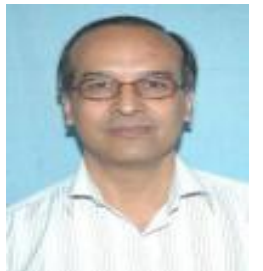

Vidya Prasad Shukla was born in India, in 1954. He received his M.Sc. (Applied Mathematics) in 1976, Ph.D. (Modelling and Computer Simulation) in 1982 and PG Dip. (Computational Hydraulic Engineering) in 1986 from Avadh University Faizabad, Indian Institute of Technology Kanpur and International Institute of Environmental \& Hudraulic Engineering (Delft) the Netherlands respectively. He worked and officiated at various posts as Senior Research Officer, Chief Research Officer and HOD Computer Division at from Central Water and Power research Station (CWPRS), Pune from 1982 to 2003. Thereafter, he worked as a Professor in BIT, Sathyamangalam and NIT Durgapur. He has joined as a Professor in Mody Institute of Technology \& Science, Deemed University Laxmangarh in 2009. He has published over 57 papers in refereed journals and conference proceedings and written 29 technical reports on various clients sponsored research projects of international/national importance. He is an editor of the book "Development of Coastal Engineering" from CWPRS, Pune. His current research interest includes Computer Simulation \& Modelling, Image processing, Cellular Automata, SoftComputing, Computer Vision, Nanotech-simulation, Operations Research, Mathematical Biology, Modelling of Arms Race of Nations.

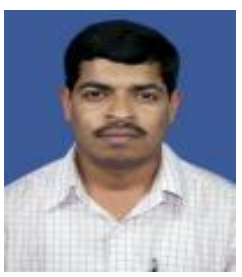

Mr. S.R. Biradar is a Professor in the department of Computer Science and Engineering, SDM, Dharwad, India. He received his B.E, M.Tech and Ph.D. degrees in Computer Science and Engineering from Karnataka University, MAHE Manipal and Jadavpur University respectively. His research interest includes Mobile Ad-hoc networking, advanced wireless communication. He has published over 45 papers in refereed journals and conference proceedings. His current research interest includes Image Processing, Mobile ad hoc networks, and sensor networks.

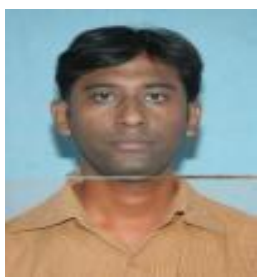

Ajay Kumar Singh was born in India, in 1980 . He received his B.E. (Computer Sc. \& Engineering) in 2001, M.Tech. (Information Technology) in 2006 from CCS University Meerut and AAI Deemed University Allahabad respectively. He has joined as an Asst. Prof. in Mody Institute of Technology \& Science, Deemed University Laxmangarh in 2009. Presently, he is pursuing Ph.D. in Computer Sc. \& Engg. from the MITS Lakshmangarh. He has published over 20 papers in refereed journals and conference proceedings. His current research interest includes Image Processing, Image classification and their applications in computer vision.

How to cite this paper: Shamik Tiwari, V. P. Shukla, S. R. Biradar, A. K. Singh,"Blur Classification Using Wavelet Transform and Feed Forward Neural Network", IJMECS, vol.6, no.4, pp.16-23, 2014.DOI: 10.5815/ijmecs.2014.04.03 